\title{
The BEST Dataset of Language Proficiency
}

\author{
Angela de Bruin ${ }^{1 *}$, Manuel Carreiras ${ }^{1,2,3}$ and Jon Andoni Duñabeitia ${ }^{1}$ \\ 1 Basque Center on Cognition, Brain and Language, Donostia, Spain, ${ }^{2}$ Departamento de Lengua Vasca y Comunicación, \\ University of the Basque Country, Bilbao, Spain, ${ }^{3}$ Ikerbasque, Basque Foundation for Science, Bilbao, Spain
}

Keywords: Basque language, Spanish language, English language, proficiency test, multilingualism, language skills, Basque Country

\section{INTRODUCTION}

Researchers investigating processes stemming from or related to multilingualism often face the challenge of correctly characterizing their multilingual samples in terms of language use, proficiency, dominance, and exposure. This is typically done by using a variety of objective (e.g., normed tests) and/or subjective (e.g., self-reports) measures which tend to vary largely across laboratories and studies, since to date the existence of a comprehensive set of measures and norms is limited to certain language combinations (see Gollan et al., 2012). The current study provides a compelling dataset of norms obtained from a large number of Spanish-Basque-English multilinguals from the Basque Country that will facilitate participant selection and classification as a function of their background and language skills.

Contrary to Spanish and English, the Basque language is not a member of the Indo-European language family. It is widely used within the autonomous communities of the Basque Country and Navarra, both located in northern Spain, as well as in the French Pyrénées-Atlantiques. The current study focuses on multilinguals living in the Spanish autonomous community of the Basque Country, an area containing a wealth of bilingual speakers of Spanish and Basque, the two co-official languages, who also know English as a foreign language to a certain extent. Many Basque adults grew up speaking Basque and/or Spanish at home and subsequently received education in one or both of these languages. Additionally, English has been taught as part of the Spanish academic curriculum from the early 1970s, and nearly everyone in the Basque Country below the age of 50 has been exposed to English at school. For this reason, the majority of young and middle-aged Basque adults are better characterized as multilinguals than as bilinguals solely. However, this multilingual population's knowledge of languages is not homogeneous. While some multilinguals are Spanish dominant, others use Spanish and Basque in a more balanced way or are more dominant in Basque. Furthermore, the exposure to and proficiency in English varies greatly.

The complex linguistic reality of the Basque Country provides researchers with an ideal environment to investigate aspects related to multilingualism. For this reason, recent years have witnessed an exponential increase in the number of psycholinguistic studies on Basque bi-/multilinguals exploring semantic (e.g., Perea et al., 2008), syntactic (e.g., Díaz et al., 2016), lexical (e.g., Duñabeitia et al., 2010b), and ortho-phonological processes (e.g., Casaponsa et al., 2015). Besides, recent studies have also focused on Basque multilinguals in order to explore language-mediated domain-general cognitive processes such as attention (e.g., see Antón et al., 2014, 2016; Duñabeitia et al., 2014) or learning (e.g., Cenoz, 1998). Given this increased presence of studies with Basque multilinguals, some efforts have been made to provide researchers with databases that allow for the creation of adequately characterized research materials (e.g., Perea et al., 2006; Duñabeitia et al., 2010a; Acha et al., 2014). 
In order to study Basque multilinguals, and over and above creating adequately controlled materials, one also needs to characterize the different types of participants that constitute the test samples. In the current Data Report we introduce the BEST dataset, which presents data from 650 adult participants from the Basque Country who completed a series of Basque, English, and Spanish tests (hence the name BEST) as a proxy for measuring their language skills. The BEST dataset is the result of a collaborative project developed at the Basque Center on Cognition, Brain and Language (BCBL) with the aim of providing researchers from the Basque Country with a series of norms that can be used to better characterize the test samples. Here we present both the range of scores per task, the quantile distribution of these scores, as well as a cluster analysis grouping participants according to the different measurements. This database and the associated materials that include various objective and subjective measures commonly used in psycholinguistic research can be used for future studies aiming to test multilingual samples from the Basque Country.

Self-ratings are an easy and often-used method to assess participants' proficiency level. Although self-ratings have been found to correlate with more objective proficiency measures (see Marian et al., 2007), they have also been criticized, as participants may over- or underestimate their own proficiency (e.g., MacIntyre et al., 1997). Thus, self-ratings should not be taken as the unique index of language use and proficiency, although they provide useful supplementary information (see Gollan et al., 2012). For this reason, our dataset avoids the subjectivity of only using self-assessed proficiency measurements by combining a range of objective and subjective proficiency measurements. Using multiple tasks to assess proficiency offers a more comprehensive understanding of the participants' actual language proficiency.

The BEST dataset includes information from four different subtests divided in three language blocks. We report two objective measures that cover different aspects related to vocabulary knowledge, word production and visual word identification. Firstly, following the idea of the Multilingual Naming Test (Gollan et al., 2012), we designed a vocabulary test that was completed by the participants in the three languages. Secondly, participants also completed a series of lexical decision tests (one per language) similar to the LexTALE created by Lemhöfer and Broersma (2012). They had to decide whether each letter string corresponded to an existing word in the target language or not. Thirdly, and following the recommendations of Gollan et al. (2012), all participants underwent a short semistructured interview guided by a multilingual linguist with experience in assessing language proficiency who provided a score of the participants' language skills in each language. And lastly, participants completed a short questionnaire about language history and knowledge adapted from other questionnaires previously used in the literature (e.g., Marian et al., 2007). Below we present detailed information about these tests, which can be accessed together with the normative data via https://figshare.com/s/2b377367585a7e5353fb and http://hdl.handle.net/10810/20563.

\section{PARTICIPANTS}

A group of 650 (435 female) participants completed various language proficiency measurements. Their ages ranged from 18 to 50 years (mean $=25.02, S D=5.58$ ). The maximum level of education achieved at the time of testing ranged from high school to university, although the majority of participants $(80 \%)$ reached a higher level of education (professional training, university, or a postgraduate degree). All participants were Spanish-Basque-English trilinguals and they had acquired Basque and Spanish before the age of six (mean AoA $A_{\text {Spanish }}=0.67$, $S D=1.55$; mean AoA $\left.A_{\text {Basque }}=1.68, S D=1.81\right)$. On average, English was acquired at a later age (mean AoA $A_{\text {English }}=6.37$, $S D=2.49$ ), but all participants reported having acquired English at or before age 12. Regarding the dialectal variations of Basque, the majority of participants reported using either the standard Batua Basque form (54\%) or the Gipuzkoan dialect corresponding to the region in which the current study was conducted (38\%). An additional six percent of participants reported using a Biscayan or Western dialect while $1 \%$ used an upper Navarrese dialect. Although more participants took part in some of the tasks, we only included participants who completed all measurements in the final BEST dataset.

\section{TASKS AND PROCEDURE}

Data were collected over a period of 18 months, starting from January 2015 and ending in June 2016. Participants first registered and completed the questionnaire aimed at gathering the subjective measurements and the LexTALE tests using the online platform created for this purpose ${ }^{1}$. After this, they came to the laboratories where they individually completed the picturenaming tests and underwent the semi-structured interview. All participants provided signed consent forms prior to completing the battery of tests, which had been previously validated by the BCBL Ethics Committee. The materials for all tasks can be found in the public repository deposits ${ }^{2,3}$.

\section{Self-rated Proficiency and Exposure}

Self-rated proficiency and exposure scores were collected as part of an abridged version of the Language Experience and Proficiency Questionnaire (Marian et al., 2007). Participants were asked to rate their proficiency in Spanish, Basque, and English on a scale from 0 ('lowest level') to 10 ('native or native-like level') at the general level. Similarly, participants rated their estimated percentage of exposure to each of the three languages on a scale from 0 ('never') to 100 ('always').

\section{Interview}

Participants completed a short semi-structured oral proficiency interview in each of their three languages (cf., Gollan et al., 2012).

\footnotetext{
${ }^{1}$ http://www.bcbl.eu/participa/

${ }^{2}$ https://figshare.com/s/2b377367585a7e5353fb

${ }^{3}$ http://hdl.handle.net/10810/20563
} 
This 5-min interview consisted of a set of questions ranging in difficulty and requiring the participant to use different types of grammatical constructions (e.g., questions requiring different tenses). The interview was conducted and assessed by a group of linguists who were native speakers of Basque and Spanish with high proficiency in English. One linguist evaluated each participant, but a total of four linguists with previous professional experience in assessing linguistic competence took part in the process. The scoring was based on a Likert-like scale from 1 ('lowest level') to 5 ('native or native-like level').

\section{Picture Naming}

Expressive vocabulary was assessed through a picture-naming task akin to the Multilingual Naming Test (cf., Gollan et al., 2012) but specifically adapted for the three examined languages (Spanish, Basque, and English). The test consisted of 65 pictures corresponding to non-cognate words that had to be named in each of the three languages. All pictures showed common entities belonging to different categories such as animals (24 items) or body parts ( 8 items). Participants took approximately $10 \mathrm{~min}$ to complete each language version, and the score per language ranged from 0 to 65 . The pictures were taken from the MultiPic database (Duñabeitia et al., 2017). The order of the picture naming tasks was Spanish-Basque-English.

\section{LexTALE}

All participants completed three online versions of LexTALE, a short lexical decision test that has been shown to provide good estimates of language knowledge (cf. Lemhöfer and Broersma, 2012). The order of the LexTALE tasks was Spanish-BasqueEnglish. In the English version of the test, participants were presented with 60 items (40 words, 20 non-words) and they were asked to click on the corresponding button to indicate whether the item was an existing English word or not. For the Spanish version, we used LexTALE-Esp (Izura et al., 2014), which presents participants with 60 real Spanish words and 30 non-words and follows the same rationale and procedure as the original English version. A Basque version of LexTALE was developed for the same purposes following the same validation process described in the original studies. The Basque LexTALE was created in collaboration with three linguists and includes several relatively difficult non-words in order to increase the diagnostic power. While the inclusion of such non-words is restricted to few instances in the English and Spanish versions, we decided to include several exemplars after piloting the Basque version with a larger set of items and using point-biserial correlation analyses to exclude items with low diagnostic power. The final version of the Basque LexTALE comprised 75 items (50 real Basque words and 25 non-words). Thus, the ratio of words versus non-words was kept consistent across the three languages. Some items in the LexTALE tests can furthermore be considered (non-identical) cognates with the other two languages. Test scores for the three versions of the test are based on the percentages of accurate responses to words and non-words, corrected for the unequal number of words and non-words in the test. Hence, the final score in each language resulted from averaging the percentages of correct responses separately obtained for words and non-words, and is provided in terms of percentages.

\section{DATASET OVERVIEW AND DESCRIPTION}

The complete BEST dataset with the raw data per participant and task is available at https://figshare.com/s/2b377367585a7e5353fb and http://hdl.handle.net/10810/20563 in a tab-delimited plain text format and in a Microsoft Excel ${ }^{\circledR}$ spreadsheet. The files contain background information about the participants' age, gender, maximum education level, and handedness. It furthermore provides the individual values of the self-rated percentage of exposure to each language (from 0 to 100), their self-rated general proficiency (from 0 to 10 ), the scores resulting from the interviews (from 1 to 5), the number of correctly named items in the picture-naming tests (from 0 to 65), and the scores in the three LexTALE tests (from 0 to 100). The summary of these pieces of information is provided in the violin plots presented in Figure 1. Besides, Table 1 presents the information of the cutoff values for the most representative quantiles of the different variables from the 1st to the 99th percentile in steps of 5 .

A clustering procedure using all diagnostic indices (i.e., interview, self-perceived proficiency, LexTALE tests and picturenaming tests) was carried out in order to determine the potential subgroups of people considering their English and Basque linguistic skills. As Spanish proficiency was close to or at ceiling for all participants (see Figure 1), only English and Basque scores were included in the clustering analysis. K-means was used as a partitioning method for splitting the whole scaled dataset in different clusters. After inspection of the data, and according to the majority rule (namely, the highest number of indices proposing a clustering solution), the best number of clusters was set to 2 , indicating that the whole set of participants could be adequately separated in two main subgroups (see Supplementary Figure 1).

Interestingly, the general 2-clusters classification was in agreement with the individual results of parallel clustering procedures carried out on each specific index separately, as shown by the relatively high agreement values obtained (Cohen's kappa with interview $=0.529$; Cohen's kappa with self-perceived proficiency $=0.440$; Cohen's kappa with LexTALE $=0.479$; Cohen's kappa with picture-naming test $=0.565$ ). However, while there was a relatively high agreement with the individual indices, the results also suggest that the clustering method based on the four measurements was an optimal solution improving any clustering solely based on one individual measurement. In fact, the level of convergence among the clustering solutions individually provided by each index without taking into account the whole set resulted in a mean kappa of 0.382, suggesting only fair agreement (see Landis and Koch, 1977). This result suggests that a combination of measures is preferred over an index obtained from a unitary source, in line with the conclusion of the study by Gollan et al. (2012), who advocated for the use of a multi-measure approach to better estimate multilinguals' language skills. 


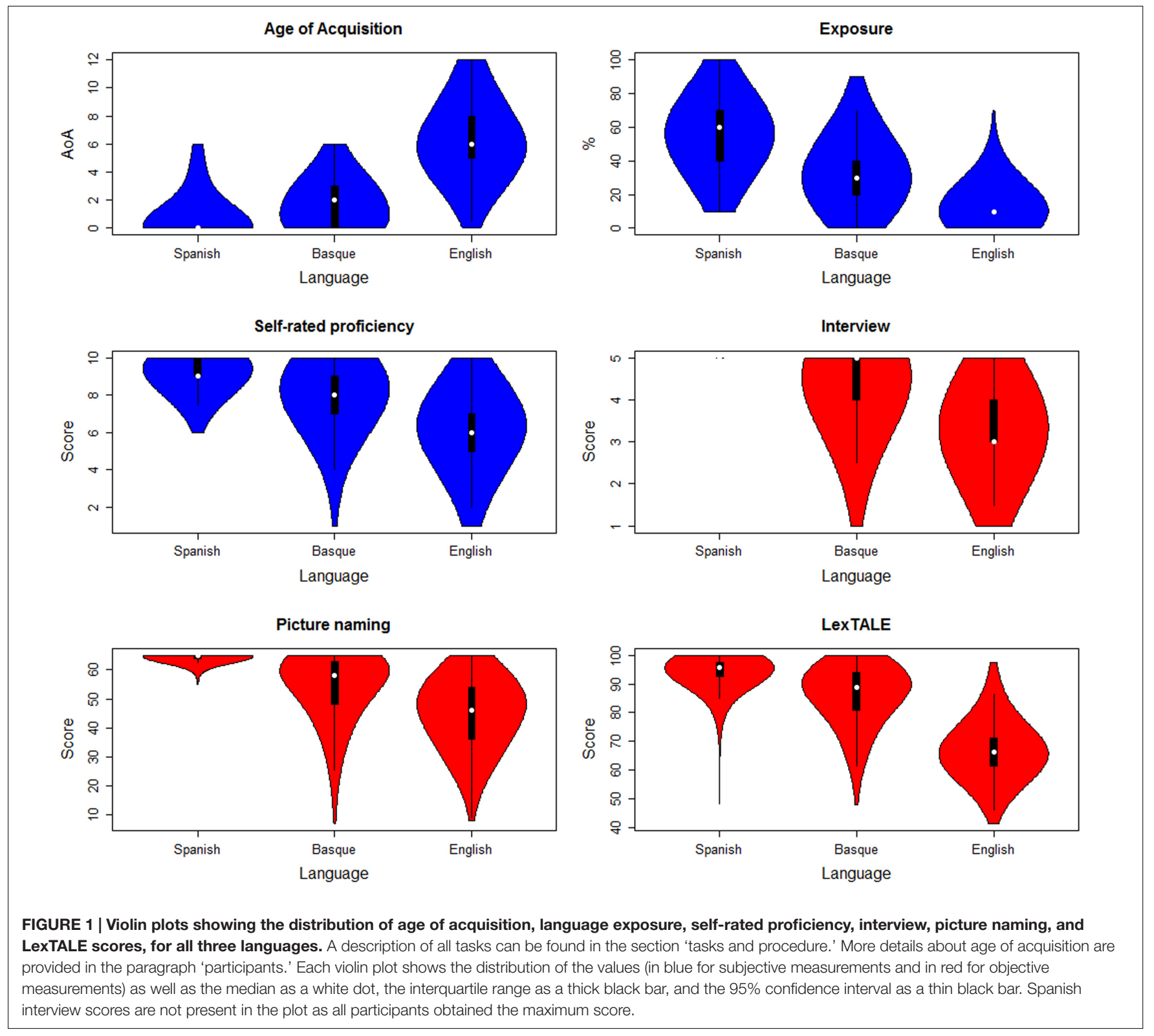

Visual inspection of the data points in the clusters and the scores on individual tests suggests that Cluster 1 (in red in Supplementary Figure 1) comprises multilinguals with an overall medium-to-high level of Basque proficiency combined with an English proficiency level ranging from low to high. Cluster 2 (in blue in Supplementary Figure 1) encompasses participants with low-to-medium Basque proficiency, regardless of their English proficiency, which varied from low to high. Thus, the division in clusters is largely based on Basque proficiency with a wide range of English proficiency in both clusters. This is not a surprising finding, given that the age of acquisition of Basque was earlier than the age of acquisition of English, and that Basque is a contextually present language in the Basque Country while English is a foreign language whose presence is mainly restricted to academic contexts.
The usefulness of a multi-dimensional battery is also selfevident when considering the correlations between the objective and subjective proficiency measurements (see Supplementary Figure 2 for correlations between all proficiency measurements as well as correlations between age of acquisition, exposure, and proficiency per language). Regarding AoA, only Basque and Spanish but not English scores correlated significantly with proficiency. In terms of proficiency measurements in Basque, all correlations ranged between $r=0.55$ (self-rated and LexTALE) and $r=0.82$ (interview and picture-naming). For English, correlations followed the same pattern, although they were more modest, ranging between $r=0.30$ (selfrated and LexTALE) and $r=0.73$ (interview and picturenaming). Again, these results demonstrate that one measure is not enough to capture the idiosyncrasy of the complexity of 


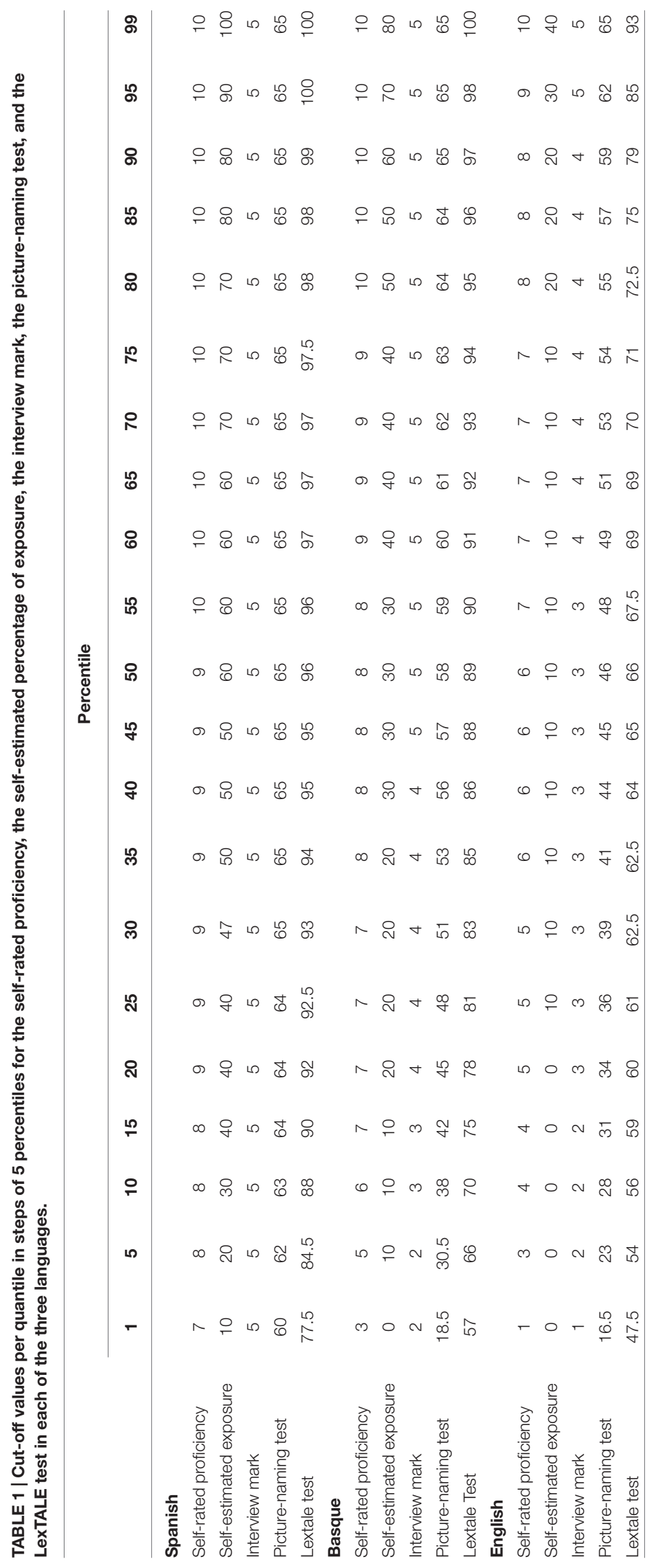


multilingualism, and they make a plea for a multi-dimensional approach.

\section{CONCLUSION}

Summarizing, the BEST dataset consists of the individual scores from a large group of multilinguals from the Basque Country who completed language proficiency measurements in Spanish, Basque, and English. While little variety was observed for the different indices of Spanish proficiency, participants showed a wide range of proficiency scores for Basque and English. Our cluster analysis showed that this multilingual group could be divided into two main subgroups mainly based on their Basque proficiency (those with low-to-medium proficiency and those with medium-to-high proficiency). Most importantly, our data highlight the importance of using multiple proficiency measurements rather than a single index from a unique test. We found relatively high agreement between the division of participants in clusters as a function of the clustering based on the four measurements and the division of participants in clusters based on each of the individual tests. In contrast, agreement between the divisions in clusters based on each one of the four individual measurements was much lower. Similarly, the correlations between the different tests in each language showed that despite the underlying common aim, the indices provided are complementary and that the additional information provided by each of them is necessary. In line with previous research, some tests correlated quite highly (e.g., Ferré and Brysbaert, 2016), but it is worth noting that none of the correlations were close to ceiling. Furthermore, correlations between self-ratings and some of the objective tests were relatively low, suggesting that self-ratings alone are not an optimal reflection of proficiency (cf. MacIntyre et al., 1997). Hence, taking multiple objective and subjective indices together provides a more complete understanding of the participants' language proficiencies.

In conclusion, the BEST dataset offers a partial snapshot of the linguistic reality of the Basque Country and these normative data can be used for a better understanding and characterization of the language knowledge and background of multilingual people from this region with a relatively high level of education.

\section{REFERENCES}

Acha, J., Laka, I., Landa, J., and Salaburu, P. (2014). EHME: a new word database for research in Basque language. Span. J. Psychol. 17:E79. doi: 10.1017/sjp. 2014.79

Antón, E., Duñabeitia, J. A., Estévez, A., Hernández, J. A., Castillo, A., Fuentes, L. J., et al. (2014). Is there a bilingual advantage in the ANT task? Evidence from children. Front. Psychol. 5:398. doi: 10.3389/fpsyg.2014.00398

Antón, E., Fernández-García, Y., Carreiras, M., and Duñabeitia, J. A. (2016). Does bilingualism shape inhibitory control in the elderly? J. Mem. Lang. 90, 147-160. doi: 10.1016/j.jml.2016.04.007

Casaponsa, A., Carreiras, M., and Duñabeitia, J. A. (2015). How do bilinguals identify the language of the word they read? Brain Res. 1624, 153-166. doi: 10.1016/j.brainres.2015.07.035

Cenoz, J. (1998). "Multilingual education in the Basque country," in Beyond Bilingualism: Multilingualism and Multilingual Education, eds J. Cenoz and F. Genesee (Clevedon: Multilingual Matters), 175-191.
The heterogeneity of the large sample tested allows for a good estimation of the language skills in one or several of the three languages explored, regardless of the number of languages known and their level of proficiency in each of them. Furthermore, our analyses show the importance of combining multiple measurements to obtain a more comprehensive understanding of language proficiency.

\section{ETHICS STATEMENT}

This study was carried out in accordance with the recommendations of $\mathrm{BCBL}$ Ethics Committee with written informed consent from all subjects. All subjects gave written informed consent in accordance with the Declaration of Helsinki. The protocol was approved by the BCBL Ethics Committee.

\section{AUTHOR CONTRIBUTIONS}

JA and MC developed the idea together and coordinated the data acquisition. AdB and JA analyzed the data. AdB drafted the manuscript and all authors approved the final version after discussing the intellectual content. All authors agreed to be accountable for all aspects of the work.

\section{FUNDING}

This research has been partially funded by grants PSI201565689-P, PSI2015-67353-R, and SEV-2015-0490 from the Spanish Government, AThEME-613465 from the European Union and a personal fellowship given by the BBVA Foundation to the last author.

\section{SUPPLEMENTARY MATERIAL}

The Supplementary Material for this article can be found online at: http://journal.frontiersin.org/article/10.3389/fpsyg. 2017.00522/full\#supplementary-material

Díaz, B., Erdocia, K., de Menezes, R. F., Mueller, J. L., Sebastian-Galles, N., and Laka, I. (2016). Electrophysiological correlates of second-language syntactic processes are related to native and second language distance regardless of age of acquisition. Front. Psychol. 7:133. doi: 10.3389/fpsyg.2016. 00133

Duñabeitia, J. A., Cholin, J., Corral, J., Perea, M., and Carreiras, M. (2010a). SYLLABARIUM: an online application for deriving complete statistics for Basque and Spanish orthographic syllables. Behav. Res. Methods 42, 118-125. doi: 10.3758/BRM.42.1.118

Duñabeitia, J. A., Crepaldi, D., Meyer, A., New, B., Pliatsikas, C., Smolka, E., et al. (2017). MultiPic: a standardized set of 750 drawings with norms for six European languages. Q. J. Exp. Psychol. doi: 10.1080/17470218.2017.1310261 [Epub ahead of print].

Duñabeitia, J. A., Dimitropoulou, M., Uribe-Etxebarria, O., Laka, I., and Carreiras, M. (2010b). Electrophysiological correlates of the masked translation priming effect with highly proficient simultaneous bilinguals. Brain Res. 1359, 142-154. doi: 10.1016/j.brainres.2010.08.066 
Duñabeitia, J. A., Hernández, J. A., Antón, E., Macizo, P., Estévez, A., Fuentes, L. J., et al. (2014). The inhibitory advantage in bilingual children revisited: myth or reality? Exp. Psychol. 61, 234-251. doi: 10.1027/1618-3169/a000243

Ferré, P., and Brysbaert, M. (2016). Can Lextale-Esp discriminate between groups of highly proficient Catalan-Spanish bilinguals with different language dominances? Behav. Res. Methods doi: 10.3758/s13428-016-0728-y [Epub ahead of print].

Gollan, T. H., Weissberger, G. H., Runnqvist, E., Montoya, R. I., and Cera, C. M. (2012). Self-ratings of spoken language dominance: a multi-lingual naming test (MINT) and preliminary norms for young and aging SpanishEnglish bilinguals. Bilingualism 15, 594-615. doi: 10.1017/S1366728911 000332

Izura, C., Cuetos, F., and Brysbaert, M. (2014). Lextale-Esp: a test to rapidly and efficiently assess the Spanish vocabulary size. Psicológica 35, 49-66.

Landis, J. R., and Koch, G. G. (1977). The measurement of observer agreement for categorical data. Biometrics 33, 159-174. doi: 10.2307/2529310

Lemhöfer, K., and Broersma, M. (2012). Introducing LexTALE: a quick and valid lexical test for advanced learners of English. Behav. Res. Methods 44, 325-343. doi: 10.3758/s13428-011-0146-0

MacIntyre, P. D., Noels, K. A., and Clément, R. (1997). Biases in self-ratings of second language proficiency: the role of language anxiety. Lang. Learn. 47, 265-287. doi: 10.1111/0023-8333.81997008
Marian, V., Blumenfeld, H. K., and Kaushanskaya, M. (2007). The Language Experience and Proficiency Questionnaire (LEAP-Q): assessing language profiles in bilinguals and multilinguals. J. Speech Lang. Hear Res. 50, 940-967. doi: 10.1044/1092-4388(2007/067)

Perea, M., Duñabeitia, J. A., and Carreiras, M. (2008). Masked associative/semantic and identity priming effects across languages with highly proficient bilinguals. J. Mem. Lang. 58, 916-930. doi: 10.1016/j.jml.2008.01.003

Perea, M., Urkia, M., Davis, C. J., Agirre, A., Laseka, E., and Carreiras, M. (2006). E-Hitz: a word-frequency list and a program for deriving psycholinguistic statistics in an agglutinative language (Basque). Behav. Res. Methods 38, 610-615. doi: 10.3758/BF03193893

Conflict of Interest Statement: The authors declare that the research was conducted in the absence of any commercial or financial relationships that could be construed as a potential conflict of interest.

Copyright $\odot 2017$ de Bruin, Carreiras and Dunabeitia. This is an open-access article distributed under the terms of the Creative Commons Attribution License (CC BY). The use, distribution or reproduction in other forums is permitted, provided the original author(s) or licensor are credited and that the original publication in this journal is cited, in accordance with accepted academic practice. No use, distribution or reproduction is permitted which does not comply with these terms. 